\title{
EVALUACIÓN DE LA EFECTIVIDAD DEL ULTRASONIDO ABDOMINAL EN EL DIAGNÓSTICO DE ESTENOSIS HIPERTRÓFICA DE PÍLORO EN NIÑOS DEL HOSPITAL DR. ROBERTO GILBERT ELIZALDE
}

\author{
EFFECTIVENESS EVALUATION OF ABDOMINAL ULTRASOUND FOR DIAGNOSIS OF PEDIATRIC \\ HYPERTROPHIC PYLORIC STENOSIS AT DR. ROBERTO GILBERT ELIZALDE HOSPITAL
}

\author{
AVALIAÇÃO DA EFETIVIDADE DO ULTRASSOM ABDOMINAL NO DIAGNOSTICO DE ESTENOSES HIPERTRÓFICA \\ DO PILORO EM CRIANÇAS DO HOSPITAL DR. ROBERTO GILBERT ELIZALDE
}

\author{
MARÍA BETANCOURT RUIZ1,2, JIMMY PAZMIÑO ARROBA'1,2, MIGUEL SORIA ALCÍVAR CORREO1,2 \\ ${ }^{1}$ Universidad Católica de Santiago de Guayaquil, Ecuador. \\ ${ }^{2}$ Hospital Dr. Roberto Gilbert Elizalde, Guayaquil, Ecuador.
}

Resumen

Introducción: la estenosis hipertrófica de píloro es un engrosamiento anormal del musculo pilórico que produce obstrucción del vaciamiento gástrico; afecta con mayor frecuencia a la raza blanca, cuya característica clínica son los vómitos no biliosos y explosivos; su diagnóstico se realiza con el ultrasonido vs tránsito gástrico. Su tratamiento es la piloromiotomía. Objetivo: evaluar el ultrasonido abdominal (US) en el diagnóstico de la Estenosis hipertrófica del píloro (EHP) en niños del servicio de cirugía del hospital Dr. Roberto Gilbert Elizalde (HRGE), período "enero 2010-junio 2013". Materiales y métodos: estudio descriptivo, longitudinal, retrospectivo de pacientes que ingresaron al HRGE con sospecha clínica de EHP. Se analizó: características epidemiológicas, motivo de consulta, estancia hospitalaria usando promedio, desviación estándar (DS), rango; y, sensibilidad, especificidad, valor predictivo positivo y negativo del ultrasonido. Resultados: se analizaron 130 historias clínicas, 11 tenían datos incompletos. 119 pacientes ingresaron con sospecha de EHP; se confirmó el diagnóstico en 101, $87(86 \%)$ fueron varones y $14(14 \%)$ mujeres. La edad en días promedio \pm DS fue $36.97 \pm 18.24$, rango (16 a 150 ). El US tuvo una sensibilidad del $100 \%$, especificidad del $83 \%$, valor predictivo positivo del $97 \%$ y valor predictivo negativo del $100 \%$ para el diagnóstico de EHP. El principal motivo de consulta fueron los vómitos (100\% de los casos). El promedio de días de hospitalización fue $5.92 \pm 3.38$. Conclusión: el US es el mejor método diagnóstico para la EHP en los pacientes con sospecha clínica, sugiriéndose la utilización de otros estudios solo en caso de alta duda diagnóstica.

PALABRAS CLAVE: estenosis hipertrófica de píloro, vómitos, ultrasonografía.

Abstract

Introduction: hypertrophic pyloric stenosis is an abnormal thickening of the pyloric muscle that produces obstruction in gastric emptying; it most frequently affects white people. Its clinical characteristics are non-bilious and explosive vomiting; its diagnosis is made through ultrasound and/or gastric transit. Its treatment is pyloromyotomy. Objective: to evaluate abdominal ultrasound (US) in the diagnosis of pediatric hypertrophic pyloric stenosis (HPS) at the surgery service of Dr. Roberto Gilbert Elizalde Hospital (RGEH), period “January 2010-June 2013". Materials and methods: descriptive, longitudinal, retrospective study of patients admitted to the hospital because of a possible HPS. The analysis included: epidemiological characteristics, reasons for consultation, average hospital stay, standard deviation (SD), range; and sensibility, specificity, positive and negative predictive value of the ultrasound. Results: 130 clinical records were analyzed, 11 had incomplete data. 119 patients were admitted with clinical suspicion of HPS and diagnosis was confirmed in 101, 87 (86\%) were male and $14(14 \%)$ female. Average age in days $\pm \mathrm{DS}$ was $36.97 \pm 18.24$, range (16 to 150$)$. US had a sensibility of $100 \%$, specificity of $83 \%$, positive predictive value of $97 \%$ and negative predictive value of $100 \%$ in relation to the diagnosis of HPS. The principal reason for consultation was vomiting (100 \% of cases). Average hospitalization was $5.92 \pm 3.38$ days. Conclusion: US is the best diagnosis method for HPS among patients with clinical suspicion. Use of other studies is suggested just in case of high diagnostic doubts.

KEYWORDS: pyloric stenosis hypertrophic, vomiting, ultrasonography.

Resumo

Introdução: a estenoses hipertrófica do piloro é um engrossamento anormal do musculo pilórico que produz obstrução do esvaziamento gástrico; afeta com maior frequência a raça branca, cuja característica clínica são os vómitos não biliosos e explosivos; 0 seu diagnostico é realizado pelo ultrassom e/ou transito gástrico. 0 seu tratamento é a pilorometria. Objetivo: avaliar o ultrassom abdominal (US) no diagnostico das estenoses hipertróficas do piloro (EHP) em crianças do serviço de cirurgia do hospital dr. Roberto Gilbert Elizalde (HRGE), período "Janeiro 2010 - Junho 2013". Materiais e métodos: estudo descritivo, longitudinal, retrospetivo de pacientes que ingressaram ao HRGE com suspeita clínica de EHP. Foi analisado: características epidemiológicas, motivo de consulta, instancia hospitalária usando media, desvio padrão (DP), intervalo; sensibilidade, especificidade, valor preditivo positivo e negativo do ultrassom. Resultados: foram analisados 130 historias clínicas, 11 tinham dados incompletos. 119 pacientes ingressaram com suspeita de EHP; o diagnostico foi confirmado em 101, 87 (86\%) foram do sexo masculino e 14 (14\%) do sexo feminino. A idade, em dias, media $\pm \mathrm{DP}$ foi $36.97 \pm 18.24$, intervalo (16 a 150). 0 US teve uma sensibilidade do $100 \%$, especificidade do $83 \%$, valor preditivo positivo do $97 \%$ e valor preditivo negativo do $100 \%$ para o diagnostico do EHP. Os principais motivos de consulta foram os vómitos (100\% dos casos). A media de dias de hospitalização foi de $5.92 \pm 3.38$. Conclusões: o US é o melhor método diagnostico para a EHP nos pacientes com suspeita clinica, sendo sugerido a utilização de outros estudos apenas em caso de alta duvida diagnostica.

PALABRAS-CHAVE: estenose pilórica hipertrófica, vômito, ultrassonografia. 
INTRODUCCIÓN

La estenosis hipertrófica de píloro (EHP) es un engrosamiento anormal del píloro que produce obstrucción del vaciamiento gástrico. En Estados Unidos de Norteamérica se describe una prevalencia de 2 a 5 casos por cada 1000 recién nacidos vivos. Se presenta entre la segunda y octava semana de vida. La relación entre varones y mujeres es 4:1. Afecta con mayor frecuencia a pacientes de raza blanca en comparación con los niños afroamericanos o de origen asiático. ${ }^{1,2}$ No se cuenta con datos epidemiológicos en el Ecuador. La característica clínica de esta enfermedad son los vómitos no biliosos, progresivos y explosivos. ${ }^{3-6}$ A la inspección se observa la presencia de ondas peristálticas a nivel de hemiabdomen superior; a la palpación profunda de la referida zona abdominal se puede evidenciar una masa pequeña, firme y móvil de aproximadamente $2 \mathrm{~cm}$ de largo que corresponde al píloro engrosado (oliva pilórica) en el $85 \%$ de los lactantes. ${ }^{3,6-8}$ El US es considerado un método seguro, indoloro, con una sensibilidad entre 76 a $100 \%$ y una especificidad entre 85 a $100 \%{ }^{8-11}$ Ante la sospecha EHP también se deben solicitar exámenes de laboratorio para descartar la presencia de alteraciones hidroelectrolíticas y trastornos metabólicos (alcalosis metabólica);3,5,8 el tratamiento de elección es la piloromiotomía que se hace por laparotomía o laparoscopía. 5,12

En el HRGE el manejo de la EHP se basa en la guía de atención del niño portador de EHP utilizada en el hospital de niños de Pittsburgh de Estados Unidos, de Norteamérica la cual utiliza como principal método diagnóstico al US, complementándose con exámenes de laboratorio y radiológicos, por lo que se decidió realizar este trabajo de investigación considerando que los pacientes tienen distintas características epidemiológicas, genéticas y sociales, por lo que se estima importante conocer la casuística que pueda servir para la elaboración de la guía pertinente del HRGE. Las imágenes por US como se reporta en la literatura, tienen una sensibilidad y especificidad bastante variable en las diferentes series, ${ }^{2}$ La guía de Pittsburgh tiene como criterios ecográficos diagnósticos de EHP, las siguientes medidas: grosor del músculo pilórico mayor o igual a $5 \mathrm{~mm}$ y longitud del canal pilórico mayor a $17 \mathrm{~mm}$, estas dimensiones fueron tomados del trabajo de Bowen en 1988, el cual analizó las dimensiones del píloro en niños del Children's Hospital of Pittsburgh. ${ }^{13}$
El objetivo de este estudio es evaluar la efectividad del US mediante la sensibilidad, especificidad, el valor predictivo positivo y negativo en el diagnóstico de la EHP en niños que ingresan con sospecha clínica; además de describir las características epidemiológicas de estos pacientes, incluye las medidas ultrasonográficas promedio en milímetros, sus alteraciones electrolíticas y su estancia hospitalaria.

\section{MATERIALES Y MÉTODOS}

Se realizó un estudio descriptivo, longitudinal, retrospectivo, monocéntrico de pacientes que ingresaron al HRCE con sospecha clínica de EHP, período “enero 2010-Junio 2013”, a quienes se les había practicado un US con fines diagnósticos. Se analizó las características epidemiológicas, motivo de consulta, estancia hospitalaria, grosor y longitud del píloro usando: promedio, desviación estándar (DS), rango y para determinar significancia estadística $\mathrm{p}<0.05$, según corresponda; además del análisis de sensibilidad, especificidad, valor predictivo positivo y negativo del ultrasonido. También se realizó t-test para comparar grosor y longitud del píloro entre los pacientes que tuvieron diagnóstico definitivo y otro diagnóstico diferente de EHP. Después de una rigurosa recolección de datos estadísticos de los pacientes de la muestra de acuerdo a los criterios de inclusión y exclusión obtenidos del sistema informático SERVINTE y del ONBASE se ingresaron los datos recolectados en una tabla de microsoft office excel 2007 y luego se los traspaso al programa estadístico SPSS 14 para el análisis de todas las pruebas estadísticas descritas previamente.

La población de estudio estuvo conformada por todos los pacientes en edad pediátrica a partir de las 0 semanas, atendidos por el servicio de Cirugía en el HRGE y la muestra fueron todos los pacientes con sospecha clínica de EHP, a quienes se les había realizado US y que posteriormente fueron intervenidos quirúrgicamente con fines terapéuticos o fue descartada su enfermedad con otras pruebas diagnósticas. Se ha seguido las recomendaciones de la Declaración de Helsinki.

Se incluyó pacientes en edad pediátrica a partir de las 0 semanas de ambos sexos, que ingresaron con sospecha clínica de EHP y que se les realizó piloromiotomía o se descartó la enfermedad con otras pruebas diagnósticas. 
Se excluyó pacientes con registros incompletos de la historia clínica y en quienes no se haya realizado US con medición del píloro.

RESULTADOS

Se analizaron 130 historias clínicas de pacientes que ingresaron con sospecha clínica de EHP de las cuales se descartaron 11 por datos incompletos; por lo tanto se incluyeron 119 en el estudio. (Figura 1)

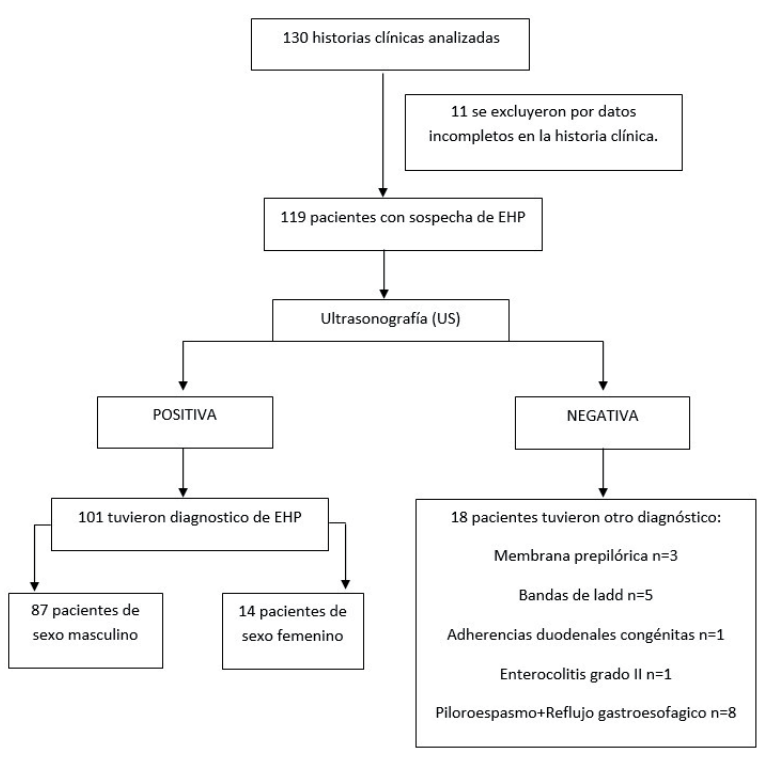

Figura 1. Diagrama de flujo del total de pacientes incluidos en el estudio con sospecha de estenosis hipertrófica de píloro del hospital de niños Dr. Roberto Gilbert Elizalde período enero 2010-junio 2013.

De 119 pacientes con sospecha de EHP 103 fueron del sexo masculino $(86 \%$ ) y del sexo femenino $16(14 \%)$. La edad en promedio en días fue con una DS de $36.77 \pm 20.37$. El rango de edad fue de 16 a 150 días. De 119 pacientes el diagnóstico de EHP fue confirmado en 101 pacientes mediante el hallazgo quirúrgico; de estos, 101 pacientes, $87(86 \%)$, fueron del sexo masculino y $14(14 \%)$ de sexo femenino. La relación en cuanto al sexo fue $6: 1$ a favor del sexo masculino.

La distribución anual de los pacientes con diagnóstico definitivo de EHP según el sexo fue la siguiente: en el 2010 se diagnosticaron 29 pacientes ( 26 de sexo masculino y 3 de sexo femenino); en el 2011 se diagnosticaron 26 pacientes (22 de sexo masculino y 4 de sexo femenino); en el 2012 se diagnosticaron 30 pacientes ( 26 de sexo masculino y 4 de sexo femenino); en el 2013 (los 6 primeros meses) se diagnosticaron 16 pacientes (13 de sexo masculino y 3 de sexo femenino).
Con base a la literatura internacional actual, se consideró como un valor inequívoco de normalidad del píloro en el US, cuando tenía grosor igual o menor de $1 \mathrm{~mm}$ y la longitud igual o menor de $10 \mathrm{~mm}$, no se pudo tomar en cuenta el diámetro, puesto que en el HRGE, no se utilizó como medida durante la realización del US, es decir valores por encima de los descritos se consideraron como US positivo.

Del total de 119 pacientes, 101 cuyo diagnóstico fue EHP, evidenciado mediante el hallazgo quirúrgico, todos tuvieron un valor de ultrasonido positivo. 18 pacientes tuvieron un diagnóstico negativo de EHP; en 3 de ellos tuvieron valores de US positivos para EHP y 15 tuvieron valores negativos al US. Del grupo de US negativo en total se evidenciaron 15 pacientes: se encontró Bandas de ladd en 5 casos (todos de sexo masculino), adherencias duodenales congénitas en 1 caso (se sexo masculino) enterocolitis grado II en 1 caso ; piloroespasmo asociado a reflujo gastroesofágico severo en 8 casos ( 7 de sexo masculino y 1 de sexo femenino). Los pacientes con bandas de ladd y adherencias duodenales fueron evidenciados en el tiempo intraoperatorio, mientras que el resto de patologías se complementaron con tránsito esofagogastrointestinal.

Se analizó mediante una tabla los valores de sensibilidad, especificidad, valor predictivo positivo y negativo del US del total de paciente que ingresaron con sospecha clínica de EHP con sus respectivos intervalos de confianza. (Tabla 1).

TABLA 1. SENSIBILIDAD, ESPECIFICIDAD, VALOR PREDICTIVO POSITIVO Y NEGATIVO CON INTERVALO DE CONFIANZA DEL ULTRASONIDO ABDOMINAL EN PACIENTES CON SOSPECHA DIAGNÓSTICA DE ESTENOSIS HIPERTRÓFICA DE PÍLORO DEL HOSPITAL DR. ROBERTO GILBERT ELIZALDE PERÍODO ENERO 2010-JUNIO 2013

\begin{tabular}{|c|c|c|c|}
\hline \multirow{2}{*}{ Test positivo (US) } & \multicolumn{3}{|c|}{$\begin{array}{r}\text { OTRAS } \\
\text { ENFERMEDADES }\end{array}$} \\
\hline & 101 & 3 & 104 \\
\hline \multirow[t]{2}{*}{ Test negativo (US) } & 0 & 15 & 15 \\
\hline & 101 & 18 & 119 \\
\hline \multicolumn{4}{|c|}{ Sensibilidad 100\% IC 95\% (100-100) } \\
\hline \multicolumn{4}{|c|}{ Especificidad 83\% IC 95\% (66.1-100) } \\
\hline \multicolumn{4}{|c|}{ Valor predictivo positivo 97\% IC 95\% (93.9-100) } \\
\hline \multicolumn{4}{|c|}{ Valor predictivo negativo 100\% IC 95\% (100-100) } \\
\hline \multicolumn{4}{|c|}{$\begin{array}{l}\text { La ultrasonografía tiene una alta sensibilidad (100\%) y Valor predictivo } \\
\text { negativo (100\%) en los pacientes con diagnóstico definitivo de Estenosis } \\
\text { hipertrófica de píloro. }\end{array}$} \\
\hline
\end{tabular}


El promedio del grosor del píloro encontrado fue de $4.55 \mathrm{~mm}$ con una desviación estándar de $\pm 1.46 \mathrm{~mm}$ de los pacientes con diagnóstico intraoperatorio de EHP, la moda fue de $4 \mathrm{~mm}$, y su rango fue de $1.9 \mathrm{~mm}$ a $13 \mathrm{~mm}$; se realizó además cálculo de cuartiles; el primer cuartil fue de $3.8 \mathrm{~mm}$ y el tercer cuartil fue de 5 , la distribución total se graficó mediante diagrama de caja o blox spot. (Figura 2).

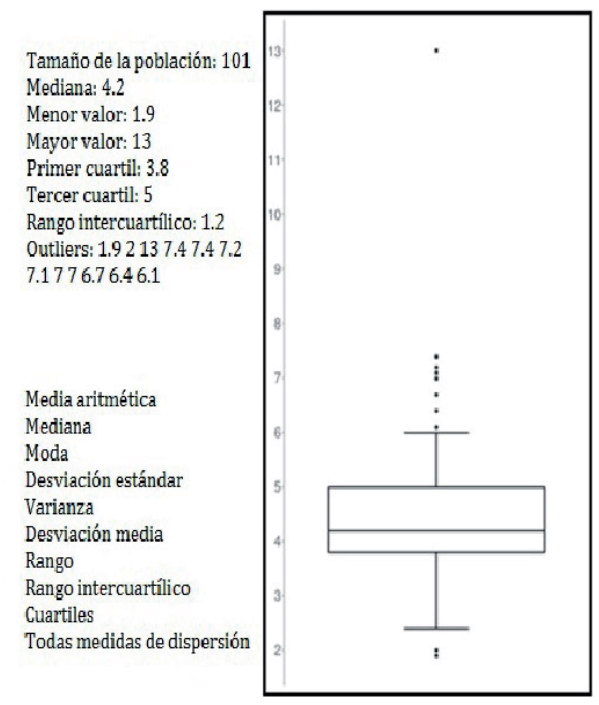

Figura 2. Diagrama de caja del grosor del píloro en milímetros de los pacientes ingresados con diagnóstico definitivo de Estenosis hipertrófica de píloro en el hospital Dr. Roberto Gilbert Elizalde período enero 2010-junio 2013.

El promedio de la longitud del píloro fue de $18.64 \mathrm{~mm}$ con una desviación estándar de \pm 3.61 de los pacientes con diagnóstico intraoperatorio del EHP, la moda fue de $17 \mathrm{~mm}$, su rango fue de $12 \mathrm{~mm}$ a $31 \mathrm{~mm}$; se realizó además cálculo de cuartiles; el primer cuartil fue de $16 \mathrm{~mm}$ y el tercer cuartil fue de 20, la distribución total se graficó en diagrama de caja o blox spot. (Figura 3).

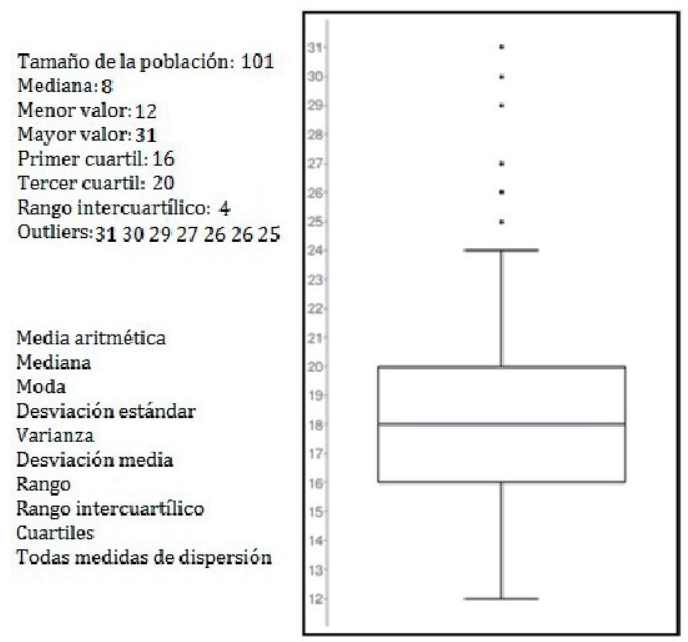

Figura 3. Diagrama de caja de la longitud del píloro en milímetros de los pacientes ingresados con diagnóstico definitivo de Estenosis hipertrófica de píloro en el hospital Dr. Roberto Gilbert Elizalde período enero 2010-junio 2013
Se realizó un t-test entre los pacientes con sospecha de EHP que tuvieron US positivo y US negativo, encontrándose una diferencia significativamente estadística entre los dos grupos, p: $<0.0001$. (Tabla 2)

\begin{tabular}{|c|c|c|c|}
\hline $\begin{array}{l}\text { MEDIDAS EN } \\
\text { MILIMETROS }\end{array}$ & $\begin{array}{r}\text { ECOGRAFIA } \\
\text { POSITIVA (MEDIA) }\end{array}$ & $\begin{array}{r}\text { ECOGRAFIA NEGATIVA } \\
\text { (MEDIA) }\end{array}$ & T-TEST \\
\hline Grosor & $4.55 \mathrm{~mm} \pm 1.46$ & $2.40 \mathrm{~mm} \pm 0.60$ & $\mathrm{p}:<0.0001$ \\
\hline Longitud & $18.64 \mathrm{~mm} \pm 3.61$ & $12.11 \mathrm{~mm} \pm 1.32$ & $\mathrm{p}:<0.0001$ \\
\hline
\end{tabular}

El motivo de consulta de los pacientes que ingresaron cuyo diagnóstico definitivo fue EHP que se presentó con mayor frecuencia fueron los vómitos, seguidos de otras causas. (Figura 4).

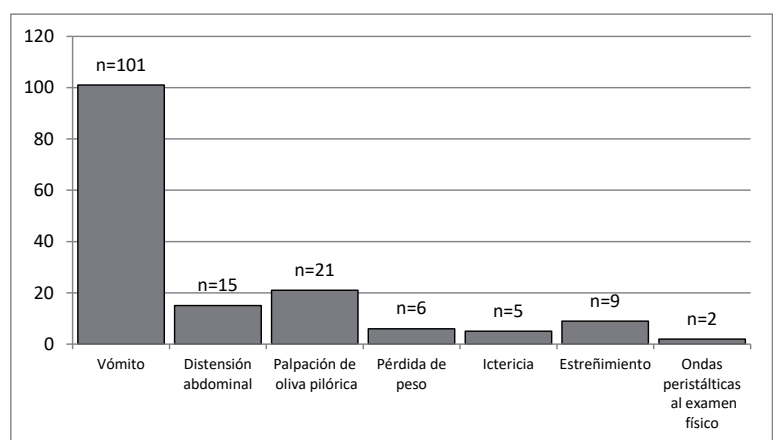

Figura 4. Motivo de consulta de los pacientes con diagnóstico definitivo de Estenosis hipertrófica de píloro en el hospital Dr. Roberto Gilbert Elizalde período enero 2010-junio 2013

Fuente: Departamento de Estadística del hospital Dr. Roberto Gilbert Elizalde.

El promedio en días con su respectiva desviación estándar de la estancia hospitalaria de todos los pacientes con diagnóstico definitivo de EHP fue $5.92+-3.38$ con un rango (3-26), moda 4.

\section{DISCUSIÓN}

Al igual que la literatura internacional en donde al EHP es más común en los pacientes de sexo masculino, ${ }^{1,2}$ en el presente estudio la relación fue de 6:1 a favor del sexo masculino; no se determinó porque es más común en los varones; esto podría estar relacionado con factores genéticos. Mientras que en los pacientes de raza blanca, es más frecuente la presentación de esta enfermedad entre la tercera a quinta semana y en los asiáticos desde la cuarta a séptima semana ${ }^{14}$; en este estudio se evidenció especialmente a los 36.97 días \pm 18.24 , con un rango de 16 días (4 semanas) a 120 días (30 semanas). 
En este país, la EHP es una enfermedad de muy baja prevalencia, en promedio fue de 7 a 8 casos por cada 100.000 habitantes, razón por la cual fue acertado realizar este estudio de forma retrospectiva; a diferencia de Norteamérica, Europa y países asiáticos donde va de 2 a 5 casos por cada 1000 habitantes en orden decreciente. , $^{1,15}$

La literatura internacional describe al US abdominal como el método complementario inicial en el diagnóstico de la EHP, bordeandouna sensibilidad entre 76 a $100 \%$ y una especificidad entre 85 a 100 $\%$ 8-11 en los resultados obtenidos en el estudio, el US con respecto a la EHP tuvo una sensibilidad del 100 $\%$ con un IC 95\% (100-100) y una especificidad del $83 \%$ con un IC $95 \%$, el valor predictivo positivo fue del $97 \%$ con un IC $95 \%$ (93-100) y el valor predictivo negativo del $100 \%$ con un IC $95 \%$ lo que lo sitúa como el principal método diagnóstico, en muchos casos evitando otros exámenes complementarios tales como el tránsito esogafogastroduodenal con los riesgos de broncoaspiración que implica el procedimiento, dejando su utilidad solamente en casos de alta duda diagnóstica.

El promedio del grosor del píloro encontrado fue de $4.55 \mathrm{~mm}$ con una DS de $\pm 1.46 \mathrm{~mm}$ de los pacientes con diagnóstico intraoperatorio del EHP, la moda fue de $4 \mathrm{~mm}$, y su rango fue de $1.9 \mathrm{~mm}$ a $13 \mathrm{~mm}$. El promedio de la longitud del píloro fue de $18.64 \mathrm{~mm}$ con una DS de \pm 3.61 de los pacientes con diagnóstico intraoperatorio del EHP, la moda fue de $17 \mathrm{~mm}$.

Este estudio aporta datos relevantes en cuanto a que estos valores, son diferentes a los que se utilizan como guía en el HRGE. El promedio del grosor del píloro fue de $4.55 \mathrm{~mm}$ con una DS de 1.46 es decir que el promedio del valor inferior para considerarlo diagnóstico es de $3.1 \mathrm{~mm}$, y si se toma en cuenta el valor de la US negativa que fue de 2.40 $\mathrm{mm} \pm 0.60$ su valor superior también es $3.1 \mathrm{~mm}$, por lo que este sería el punto de cohorte para indicar que un paciente tiene diagnóstico de EHP por US con un alto nivel de sensibilidad y especificidad. El promedio de la longitud del píloro fue de $18.64 \mathrm{~mm}$ con una DS de 3.61 por lo que el valor inferior es de $15 \mathrm{~mm}$, en cuanto al US negativo el promedio fue de $12.11 \mathrm{~mm} \pm 1.32$ por lo que su valor más superior seria $13.5 \mathrm{~mm}$ lo que nos dejaría una brecha de $1.5 \mathrm{~mm}$ entre un valor positivo y negativo, pero cuando se complementa con el grosor, mejora mucho la aproximación diagnóstica. Cabe recalcar que entre los valores descritos, se encontraron diferencias significativamente estadísticas (p: $<0.0001$ ).
Los valores obtenidos, son inferiores a la guía actual, quizás relacionados con la talla, peso y factores raciales de nuestra población, por lo que se considera que estos valores se pueden traspolar a la población pediátrica de pacientes que lleguen al HRGE con sospecha clínica de EHP.

El principal síntoma de la EHP son los vómitos, además de otros síntomas como pérdida de peso, distensión abdominal, ictericia, estreñimiento en orden decreciente. Asimismo al examen físico se suele evidenciar la oliva pilórica y ondas peristálticas en el abdomen. $\cdot^{3-6,18-20}$ En este estudio el síntoma fueron los vómitos y estuvieron presentes en el $100 \%$ de los pacientes, la principal discordancia se evidenció en la palpación de la oliva pilórica que en el presente estudio se encontró en el $21 \%$ de los casos, mientras que en la literatura internacional se puede evidenciar en el $85 \%{ }^{4,19-21}$

El promedio en días con su respectiva desviación estándar de la estancia hospitalaria de todos los pacientes con diagnóstico definitivo de EHP fue $5.92+-3.38$ con un rango. ${ }^{15,22} \mathrm{El}$ alta depende de la tolerancia oral del paciente que en lo descrito en la bibliografía es muy variable y depende de factores individuales de los pacientes. ${ }^{3,5,7}$

\section{CONCLUSIONES}

El US es el mejor método diagnóstico para la EHP en los pacientes con sospecha clínica de la enfermedad, sugiriéndose la utilización de otros exámenes complementarios solo en caso de alta duda diagnóstica.

Un grosor igual o mayor de $3.1 \mathrm{~mm}$ y/o una longitud igual o mayor de $15 \mathrm{~mm}$ del píloro determinado en la US, debe ser considerado como un valor de alta sensibilidad y especificidad que diagnostica la EHP, valores inferiores deberían ser corroborados con estudios complementarios como el transito esofagogastroduodenal.

\section{REFERENCIAS BIBLIOGRÁFICAS}

1. Georgoula C, Gardiner M. Pyloric stenosis a 100 years after Ramstedt. Arch Dis Child. 2012;97(1717):741-5.

2. Grosfeld J, Onill J, Fonkalsrud E. Pediatric Surgery. Gta ed. St. Louis: Mosby; 2006.

3. Ashcraft K, Holder T. Cirugía Pediátrica. 2da ed. México: Nueva Editorial Interamericana, S.A. de C.V; 1995.

4. Doletski S, Isakov Y. Cirugía Infantil. 1era ed. Barcelona: JIMS; 1974. 
5. Noguera-Valverde RA. Estenosis hipertrófica del píloro. Rev Cubana Pediatr. 2009;81(3):8.

6. Hilton S. Radiología Pediátrica. 1era ed. México: El Manual Moderno, S.A. de C.V; 1987.

7. Ashcraft K, Murphy J, Sharp R, Sigalet D, Snyder Ch. Cirugía Pediátrica. 3era ed. México: McgrawHill Interamericana editores, S.A. de C.V; 2000.

8. Valoria J. Cirugía Pediátrica. lera ed. España: Díaz de Santos, S.A; 1994.

9. Kirs D, Thorne G. Radiología Pediátrica. 3era ed. España: Marban; 2000.

10. Onesimo R, Giorgio V, Monaco S, Fundarò C. A crying baby: not simply infant colic. BMJ Case Rep [Internet]. 2012;2012(2):257-62. Available from: http://www.ncbi.nlm.nih.gov/pubmed/22914238

11. Teele RL, Smith EH. Ultrasound in the diagnosis of idiopathic hypertrophic stenosis. N Engl J Med. 1997;296:1449-50.

12. St Peter SD, Holcomb GW 3rd, Calkins CM, et al. Open versus laparoscopic pyloromyotomy for pyloric stenosis: A prospective, randomized trial. Ann Surg. 2006;244:363-70.

13. Bowen A. The vomiting infant: recent advances and unsettled issues in imaging. Radiol Clin North Am. 1988 Mar;26(2):377-92.

14. Leong MM, ChenSolomon SCC, Hsieh CS, Chin YY, Tok TS, Wu SF, et al. Epidemiological features of infantile hypertrophic pyloric stenosis in taiwanese children: A nation-wide analysis of cases during 1997-2007. PLoS One. 2011;6(5):1-6.

15. Pedersen RN, Garne E, Loane M, Korsholm L, Husby S. Infantile hypertrophic pyloric stenosis: a comparative study of incidence and other epidemiological characteristics in seven European regions. J Matern Fetal Neonatal Med. 2008;21(9):599-604.

16. De Laffolie J, Turial S, Heckmann M, Zimmer K-P, Schier F. Decline in Infantile Hypertrophic Pyloric Stenosis in Germany in 2000-2008. Pediatrics. 2012;129(4):e901-6.

17. Vega N, Coto G, Alvarez V, Raposo L, Rodríguez D. Cambios epidemiológicos y diagnóstico en la estenosis hipertrófica de píloro. Acta Pediatr Esp. 2013; 71(5):117-22.

18. Caffey J. Diagnóstico radiológico en pediatría. 3era ed. España: Salvat Editores S.A; 1982.

19. Demian M, Nguyen S, Emil S. Early pyloric stenosis: A case control study. Pediatr Surg Int. 2009;25(12):1053-7.

20. Villalba S, Espínola M, Ferreira M, Díaz C. Estenosis Hipertrófica Pilórica en menores de 2 meses. Rev Salud Pública Parag. 2013; 3(2):17-22.

21. Soto $M$, Arias A. Hipertrofia Congénita del Píloro. Rev. Méd. de Costa Rica y Centro América. 2008; (583): 159-62.

22. Vanderwinden JM, Liu H, De Laet MH, Vanderhaeghen JJ. Study of the interstitial cells of Cajal in infantile hypertrophic pyloric stenosis. Gastroenterology. 1996;111(2):279-88. 